\title{
Activity-Dependent Calcium Signaling in Neurons of the Medial Superior Olive during Late Postnatal Development
}

\author{
DDelwen L. Franzen, ${ }^{1,2}$ Sarah A. Gleiss, ${ }^{1,2}$ @Christian J. Kellner, ${ }^{2,3}$ Nikolaos Kladisios, ${ }^{4}$ and Felix Felmy ${ }^{1,4}$ \\ ${ }^{1}$ Division of Neurobiology, Department Biology II, ${ }^{2}$ Graduate School of Systemic Neurosciences, ${ }^{3}$ Computational Neuroscience, Department Biology II, \\ Ludiwg-Maximilians-University Munich, D-82152 Planegg-Martinsried, Germany, and ${ }^{4}$ Institute of Zoology, University for Veterinary Medicine, \\ Hannover, 30559 Hannover, Germany
}

The development of sensory circuits is partially guided by sensory experience. In the medial superior olive (MSO), these refinements generate precise coincidence detection to localize sounds in the azimuthal plane. Glycinergic inhibitory inputs to the MSO, which tune the sensitivity to interaural time differences, undergo substantial structural and functional refinements after hearing onset. Whether excitation and calcium signaling in the MSO are similarly affected by the onset of acoustic experience is unresolved. To assess the time window and mechanism of excitatory and calcium-dependent refinements during late postnatal development, we quantified EPSCs and calcium entry in MSO neurons of Mongolian gerbils of either sex raised in a normal and in an activity altered, omnidirectional white noise environment. Global dendritic calcium transients elicited by action potentials disappeared rapidly after hearing onset. Local synaptic calcium transients decreased, leaving a GluR2 lacking AMPAR-mediated influx as the only activity-dependent source in adulthood. Exposure to omnidirectional white noise accelerated the decrease in calcium entry, leaving membrane properties unaffected. Thus, sound-driven activity accelerates the excitatory refinement and shortens the period of activity-dependent calcium signaling around hearing onset. Together with earlier reports, our findings highlight that excitation, inhibition, and biophysical properties are differentially sensitive to distinct features of sensory experience.

Key words: activity dependence; calcium current; calcium influx; excitatory currents; medial superior olive; postnatal development

Significance Statement

Neurons in the medial superior olive, an ultra-fast coincidence detector for sound source localization, acquire their specialized function through refinements during late postnatal development. The refinement of inhibitory inputs that convey sensitivity to relevant interaural time differences is instructed by the experience of sound localization cues. Which cues instruct the refinement of excitatory inputs, calcium signaling, and biophysical properties is unknown. Here we demonstrate a time window for activityand calcium-dependent refinements limited to shortly after hearing onset. Exposure to omnidirectional white noise, which suppresses sound localization cues but increases overall activity, accelerates the refinement of calcium signaling and excitatory inputs without affecting biophysical membrane properties. Thus, the refinement of excitation, inhibition, and intrinsic properties is instructed by distinct cues.

\section{Introduction}

Neurons of the medial superior olive (MSO) encode interaural time differences (ITDs), a binaural cue used to localize low-

\footnotetext{
Received July 1, 2019; revised Dec. 13, 2019; accepted Jan. 9, 2020.

Author contributions: D.L.F., S.A.G., N.K., and F.F. performed research; D.L.F., S.A.G., N.K., and F.F. analyzed data; D.L.F. wrote the first draft of the paper; D.L.F., N.K., and F.F. edited the paper; C.J.K. contributed unpublished reagents/analytic tools; D.L.F. and F.F. designed research; F.F. wrote the paper.

This work was supported by the Deutsche Forschungsgemeinschaft FE789/8-1. We thank Prof. Benedikt Grothe for generous support and sharing thoughts; Prof. Christian Leibold for comments on the manuscript; Alexandra Benn, Swantje Fischer, and Claudia Schulze for help with the immunofluorescence; and Dr. Jan Grewe for work on the NixView tool to visualize the imaging data.

The authors declare no competing financial interests.

Correspondence should be addressed to Felix Felmy at felix.felmy@tiho-hannover.de.
}

frequency sounds (Grothe et al., 2010). MSO neurons undergo substantial presynaptic and postsynaptic developmental refinements, which adjust their function in the mature circuit. Late postnatal refinements include the acceleration of their voltage signaling (Magnusson et al., 2005; Scott et al., 2005; Chirila et al., 2007), driven by changes in voltage-gated ion channels (Scott et al., 2005; Khurana et al., 2012) and cell morphology (Rautenberg et al., 2009). These changes are paralleled by the elaboration and subsequent pruning of presynaptic inhibitory inputs (Werthat et

https://doi.org/10.1523/JNEUROSCI.1545-19.2020

Copyright $\odot 2020$ the authors 
al., 2008) and the development of their synaptic properties (Smith et al., 2000; Magnusson et al., 2005).

MSO neurons receive bilateral excitatory and inhibitory inputs. Inhibitory synapses refine to the soma shortly after hearing onset (postnatal day 12 [P12]) (Kapfer et al., 2002; Werthat et al., 2008). Masking relevant binaural cues with cochlear ablation or by omnidirectional white noise $(\mathrm{OWN})$ rearing prevents the reorganization of inhibitory synapses to the soma (Kapfer et al., 2002; Werthat et al., 2008) and interferes with the development of glycinergic transmission (Magnusson et al., 2005). Since exposure to OWN also affects the development of ITD tuning (Seidl and Grothe, 2005), normal acoustic experience during late postnatal development instructs inhibitory refinements required for appropriate circuit function. Whether excitation and calcium signaling in the MSO refine during development and are similarly affected by changes in the acoustic environment is unresolved. Yet, it is an important question to address how an ultra-fast coincidence detector circuit becomes wired and how the functionally relevant interplay between excitation and inhibition (Brand et al., 2002; Pecka et al., 2008; Myoga et al., 2014; Goldwyn et al., 2017) is generated during development. By using the MSO, where manipulations in the acoustic environment allow the instructive role of neuronal activity and acoustic cues to be investigated (Kapfer et al., 2002; Magnusson et al., 2005; Werthat et al., 2008), we can untangle the mechanism that drives developmental refinements.

Many developmental processes in neurons depend on calcium (Berridge, 1998; Greer and Greenberg, 2008), such as neuronal growth and synapse formation (Spitzer et al., 2000; Michaelsen and Lohmann, 2010), establishment and refinement of neuronal networks (Lohmann et al., 1998; Hirtz et al., 2012), synaptic plasticity (Feldman, 2012), and neuronal survival (Franklin and Johnson, 1992). The control of the cellular calcium levels can be developmentally regulated at various levels, for example, by the developmental modulated expression of calcium binding proteins (Lohmann and Friauf, 1996; Felmy and Schneggenburger, 2004; Bazwinsky-Wutschke et al., 2016). Therefore, it is relevant to determine activity-dependent sources of calcium influx and their endogenous calcium buffering. Calcium enters neurons in at least two ways, both of which are developmentally regulated: VGCCs and calcium-permeable glutamate receptors. Dendritic calcium signals through VGCCs elicited by back-propagating action potentials can be integrated with coincidentally activated synaptic calcium signals, an event that can lead to changes in synaptic strength (Magee and Johnston, 1997; Markram et al., 1997; Feldman, 2012; Winters and Golding, 2018) possibly associated with developmental refinements. Thus, the developmental time window during which subcellular calcium integration occurs highlights the phase of activity-dependent refinements.

Here, we assessed how calcium influx into MSO neurons through VGCCs and synaptic glutamate receptors is developmentally regulated and how sensitive this regulation is to acoustic experience. During late postnatal development, global action potential-evoked calcium transients disappeared within a few days after hearing onset, likely restricting experience-dependent refinements to this period. Only AMPAR-mediated local calcium transients could be evoked at mature stages. Gerbils raised in OWN displayed an accelerated developmental decrease in calcium signaling through synaptic receptor channels and VGCCs. Our findings raise the possibility that excitation and inhibition to the MSO are differentially sensitive to distinct features of acoustic experience but need each other for the proper adjustment of synaptic balance and functional ITD tuning.

\section{Materials and Methods}

Preparation. All experiments complied with the institutional guidelines and national and regional laws. Animal protocols were approved by the Regierung of Oberbayern (according to the Deutsches Tierschutzgesetz). Mongolian gerbils (Meriones unguiculatus) of either sex of postnatal day (P) 9-60 raised in the institute's own breeding colony were used in these experiments. Gerbils were anesthetized with isoflurane and then decapitated. Brains were removed in dissection solution containing the following (in mM): 50 or 120 sucrose, $25 \mathrm{NaCl}, 27 \mathrm{NaHCO}_{3}, 2.5 \mathrm{KCl}, 1.25$ $\mathrm{NaH}_{2} \mathrm{PO}_{4}, 3 \mathrm{MgCl}_{2}, 0.1 \mathrm{CaCl}_{2}, 25$ glucose, 0.4 ascorbic acid, 3 myoinositol, and $2 \mathrm{Na}$-pyruvate ( $\mathrm{pH}$ was 7.4 when bubbled with $95 \% \mathrm{O}_{2}$ and $5 \% \mathrm{CO}_{2}$ ), and 110 - to $200-\mu \mathrm{m}$-thick transversal (young) or horizontal (adult) slices containing the MSO were cut with a VT1200S Vibratome (Leica Microsystems). Slices were incubated for $30-45 \mathrm{~min}$ at $34.5^{\circ} \mathrm{C}$ in extracellular recording solution (same as dissection solution but with 125 $\mathrm{mm} \mathrm{NaCl}$, no sucrose, $2 \mathrm{~mm} \mathrm{CaCl}_{2}$, and $1 \mathrm{~mm} \mathrm{MgCl}_{2}$ ). All recordings were performed at near-physiological temperature $\left(34^{\circ} \mathrm{C}-36^{\circ} \mathrm{C}\right)$.

Electrophysiology. MSO neurons were visualized and imaged with a $60 \times 1$ NA objective on a BX51WI microscope (Olympus) equipped with gradient contrast illumination, and a TILL Photonics imaging system (FEI) composed of a Retiga 2000DC camera and a monochromator (Polychrome V). Recordings were performed using an EPC 10/2 amplifier (HEKA Elektronik). Data for both current-clamp and voltage-clamp recordings were acquired at $50-100 \mathrm{kHz}$ and filtered at $3 \mathrm{kHz}$. The pipette resistance ranged between 2.8 and $4 \mathrm{~m} \Omega$. Recordings with access resistances between 4.5 and $10 \mathrm{~m} \Omega$ were accepted for analysis. In currentclamp recordings, the bridge balance was set to $100 \%$ after estimation of the series resistance, which was monitored repeatedly during recordings. The series resistance during whole-cell voltage-clamp recordings was compensated to a constant residual of $2-3 \mathrm{M} \Omega$. For current-clamp experiments, the internal recording solution consisted of the following (in mM): $145 \mathrm{~K}$-gluconate, $4.5 \mathrm{KCl}, 15$ HEPES, $2 \mathrm{Mg}$-ATP, $2 \mathrm{~K}_{2}$-ATP, 0.3 $\mathrm{Na}_{2}$-GTP, $7.5 \quad \mathrm{Na}_{2}$-phosphocreatine, $30 \mu \mathrm{M}$ Oregon Green 488 BAPTA-1, and $50 \mu \mathrm{M}$ AlexaFluor-594 (pH adjusted with $\mathrm{KOH}$ to 7.4, calculated liquid junction potential: $14.8 \mathrm{mV}$ ). For voltage-clamp experiments, the internal recording solution consisted of the following (in mM): 135 Cs-gluconate, 10 HEPES, 20 tetraethylammonium chloride, 3.3 $\mathrm{MgCl}_{2}, 2 \mathrm{Na}_{2}$-ATP, $0.3 \mathrm{Na}_{2}$-GTP, $3 \mathrm{Na}_{2}$-phosphocreatine, 5 CsEGTA, $10 \mu \mathrm{M}$ ZD7288 and, in some cases, $50 \mu \mathrm{M}$ AlexaFluor-568 or AlexaFluor-594 ( $\mathrm{pH}$ adjusted with $\mathrm{CsOH}$ to 7.4 , calculated liquid junction potential: $12.7 \mathrm{mV}$ ). No correction of the liquid junction potential was performed. Inhibitory synaptic currents were blocked with $0.5 \mu \mathrm{M}$ strychnine hydrochloride and $10 \mu \mathrm{M}$ SR 95531 hydrobromide during all current- and voltage-clamp recordings. To isolate calcium signals through VGCCs, AMPARs and NMDARs were blocked with $20 \mu \mathrm{M}$ DNQX disodium salt and $50 \mu \mathrm{M}$ D-AP5 or $10 \mu \mathrm{M}$ R-CPP, respectively. Whole-cell calcium currents were isolated with the addition of $1 \mu \mathrm{M}$ TTX, 2 mм 4-Aminopyridine (4-AP), 10 mm tetraethylammonium chloride, and $50 \mu \mathrm{M} Z \mathrm{ZD} 7288$. In these experiments, the external recording solution contained $2.5 \mathrm{mM} \mathrm{CaCl}_{2}$ and $0.5 \mathrm{mM} \mathrm{MgCl}_{2}$ to increase the driving force for calcium. Slices were also incubated in these divalent ion concentrations. Calcium currents were $\mathrm{P} / \mathrm{x}$ corrected. The T-type current subtraction protocol consisted of an activation part (see Fig. $1 C$ ) in which cells were held at $-85 \mathrm{mV}$ before the step protocol, and an inactivation part (see Fig. 1D) in which cells were held at $-60 \mathrm{mV}$ before the step protocol (step size was $5 \mathrm{mV}$ ). The amplitude of T-type currents was extracted by subtracting the peak current at $-35 \mathrm{mV}$ obtained with the inactivation protocol from that of the activation protocol. No additional pharmacology was included to specifically block T-type currents.

Excitatory synaptic currents were evoked by local stimulation of either medial or lateral afferent fibers with a glass electrode $(4-5 \mathrm{M} \Omega)$ filled with recording solution. A biphasic test pulse delivered by an AM2000 stimulator of $0.2 \mathrm{~ms}$ and of $\sim 20 \mathrm{~V}$ was used to search for afferent fibers. Only in case of Figure $5 A-C$ and $G$ were single-fiber EPSCs recorded. To estimate the size of EPSCs evoked by the activation of a single fiber with minimal fiber stimulation, two brief biphasic stimulation pulses $(0.2 \mathrm{~ms})$ separated by $20 \mathrm{~ms}$ were delivered to the stimulation electrode while cells 
were held at $-60 \mathrm{mV}$. This stimulation was repeated every $5 \mathrm{~s}$, and the stimulation intensity was increased in $0.5 \mathrm{~V}$ steps until an EPSC was evoked. Single-fiber activation was indicated by a mixture of success and failure events within and between paired-pulse stimulations. AMPARmediated currents were isolated with bath application of either $50 \mu \mathrm{M}$ D-AP5 or $10 \mu \mathrm{M}$ R-CPP in addition to GABA and glycine receptor blockers. The rectification of AMPAR currents was assessed by step potentials ranging from $-70 \mathrm{mV}$ to $70 \mathrm{mV}$ in $20 \mathrm{mV}$ increments and the addition of $100 \mu \mathrm{M}$ spermine in the internal recording solution. The rectification index (RI) was calculated according to Scheuss and Bonhoeffer (2014) as follows: $\mathrm{RI}=\mathrm{I}_{50 \mathrm{mV}} /$ fit $_{50 \mathrm{mV}}$, where fit corresponds to a linear fit to the peak EPSC values in response to the first four voltage steps $(-70 \mathrm{mV}$ to $-10 \mathrm{mV}$ ). To probe for calcium-permeable AMPARs, $60 \mu \mathrm{M}$ of IEM1460 was washed in the bath. Minimal stimulation paradigms evoked responses from single fibers every $5 \mathrm{~s}$, and the EPSCs were recorded before and between 10 and $20 \mathrm{~min}$ after wash in. During wash in, a $100 \mathrm{~Hz}$ train of 20 pulses was applied every $5 \mathrm{~s}$ to open AMPARs and expose the drug binding site. For data presentation, the average of the first and the last 25 responses were compared.

Immunofluorescence and confocal microscopy. Animals were anesthetized (Narcoren, pentobarbital-sodium, $20 \mathrm{mg} / \mathrm{kg}$ ) and perfused with PBS containing $0.1 \%$ heparin and $155 \mathrm{~mm} \mathrm{NaCl}$ for $\sim 5$ min before switching the perfusion to 4\% PFA. After 20 min of perfusion, the brains were removed and postfixed overnight. Brains were washed 3 times in PBS at room temperature for $5 \mathrm{~min}$ each, and slices of $40 \mu \mathrm{m}$ thickness were taken with a VT1000S vibratome (Leica Microsystems). Standard immunofluorescence was performed on free-floating slices. The slices were washed four times in PBS at room temperature for $5 \mathrm{~min}$ each before application of blocking solution ( $0.5 \%$ Triton, $1 \%$ saponin, $0.1 \%$ $\mathrm{BSA}$ ) for $1 \mathrm{~h}$. After blocking, the slices were incubated in primary antibodies (AB) (PV 1:1000, polyclonal anti-rabbit $\mathrm{AB}$, catalog \# PV 28; CR 1:500 monoclonal anti-mouse AB, clone 6B3; CB 1:1000 polyclonal anti-rabbit AB, catalog \#CB38a, Swant; Synaptic Systems antiguinea pig, catalog \#134304; MAP-2 1:1000, polyclonal anti-chicken $\mathrm{AB}$, catalog $\# \mathrm{CH} 22103$, Neuromics) overnight at $4^{\circ} \mathrm{C}$. The specificity of the ABs directed against calcium binding proteins and VGLUT1 was verified by KO staining performed by Swant and Synaptic Systems, respectively. Slices were stained with secondary ABs conjugated with either Alexa488 (Invitrogen) or Cy3 (Dianova) and fluorescent Nissl stain (Invitrogen) at room temperature for $3 \mathrm{~h}$ and finally mounted in Vectashield medium (H-1000, Vector Laboratories; Axxora) and sealed with nail polish. Confocal scans were taken with an SP System (Leica Microsystems) with a $25 \times$ objective leading to a pixel size of $781 \mathrm{~nm}^{2}$.

Dendritic calcium imaging. The internal solution contained $30 \mu \mathrm{M}$ Oregon Green 488 BAPTA-1 to visualize calcium transients (excitation wavelength: $488 \mathrm{~nm}$ ). In the case of current-clamp recordings, $50 \mu \mathrm{M}$ AlexaFluor-594 was also included as a structural marker. In these recordings, a single image of the structural marker (excitation wavelength: 594 $\mathrm{nm}$ ) was taken after recording the calcium transient in response to a given stimulation. Images were acquired at a frame rate of $33 \mathrm{~Hz}$. Binning was set to $4 \times 4$ to increase the signal-to-noise ratio. Changes in calcium influx were recorded by acquiring a $300 \mathrm{~ms}$ baseline, after which the cell was stimulated and imaged for a further $2400 \mathrm{~ms}$. For action potentialevoked calcium signals, the following stimulations were used: 1 somatically evoked action potential and trains of 3,10 , or 25 somatically evoked action potentials $(100 \mathrm{~Hz})$ at $10 \%$ above the current threshold. A small portion of the soma was always present in the iris to ensure the imaging analysis was comparable across ages. Dendrites were imaged as far distally as possible. Only trials within 7-15 min after cell opening were considered for analysis to limit the impact of VGCC rundown. No holding current was applied at any time during the experiment. Trials that displayed failures during action potential trains or in which the image drifted substantially were excluded from further analysis. For synaptically evoked calcium signals, the stretch of dendrite that displayed calcium transients was positioned such that it optimally spanned the imaging area. The following four stimulation paradigms for evoking synaptic calcium transients were used: a single pulse, and 3,10 , or 25 pulses at $100 \mathrm{~Hz}$, presented in ascending order with an intertrial time of $10 \mathrm{~s}$. Each of these stimuli was presented at least three times. To probe the calcium influx under physiological conditions (see Fig. 6), the external $\mathrm{Ca}^{2+}$ and $\mathrm{Mg}^{2+}$ concentrations were 1.2 and $1 \mathrm{~mm}$, respectively. To record AMPAR- and NMDARmediated calcium signals under voltage-clamp conditions (see Fig. 7), the external $\mathrm{Ca}^{2+}$ and $\mathrm{Mg}^{2+}$ concentrations were 2 and $0 \mathrm{~mm}$, respectively. Finally, calcium responses through NMDARs were isolated by applying $20 \mu \mathrm{M}$ DNQX to the bath. Chemicals were purchased from Sigma-Aldrich Millipore, dyes from Thermo Fischer Scientific, and drugs from Biotrend or Tocris Bioscience.

Noise exposure. A cage was placed in a $100 \times 80 \times 80 \mathrm{~cm}^{3}$ soundattenuated box in a quiet room. A $30 \mathrm{~min}$ loop of white noise was generated with a Raspberry Pi and presented via 24 speakers: 12 low-frequency $(100 \mathrm{~Hz}$ to $12 \mathrm{kHz}$ ) and 12 high-frequency $(3.5-30 \mathrm{kHz})$ speakers, 2 of each on all 6 sides of the box. Such a broadband, omnidirectional stimulus should mask most directional cues and spatially discrete sources (Withington-Wray et al., 1990). The amplitude of the noise in the center of the cage was adjusted to $75 \mathrm{~dB}$ SPL, a level that does not cause damage to the cochlea or to auditory centers (Withington-Wray et al., 1990). Seven litters of between 4 and 7 pups were used for these experiments. The male gerbil was separated from the female and the pups $1-2 \mathrm{~d}$ before noise exposure began at P8-P9. The cage used for the noise exposure lacked a house to avoid additional reverberations. A $12 \mathrm{~h}$ light/dark cycle was set up, and the temperature and humidity inside the noise box were constantly monitored and kept at $25^{\circ} \mathrm{C}$ and $40 \%$, respectively. A humidifier ensured that the humidity never fell $<30 \%$. The mother was allowed to feed ad libidum, and fresh water was provided every few days. The mother and pups were monitored without distraction several times a day using an infrared camera. At P13, the first pup was removed from the noise box for recordings, and so forth over the following days. These experiments were approved according to the German Tierschutzgesetz (TVA 55.2-1-54-2532-224-2013).

Analysis. Electrophysiological parameters were extracted from the current and voltage responses of cells using custom-written IGOR Pro procedures (WaveMetrics) and analyzed further in Microsoft Excel and Prism. For the development of action potential properties, each parameter was obtained from the average of single action potential trials in response to a $200 \mu$ s increasing and $300 \mu$ s decreasing current ramp at $10 \%$ above the current threshold.

For the analysis of action potential-evoked calcium signals, the maximal length of visible dendrite was traced with a custom written plugin in ImageJ (code available in Zenodo, https://dx.doi.org/10.5281/zenodo. 2575542), and the data were exported in the NIX format (http://www.gnode.org/nix) for further analysis with custom-written Python scripts (code available in Zenodo, https://dx.doi.org/10.5281/zenodo.2575675). A Jupyter notebook demonstrating the analysis code is available at https://github.com/delwen/CaJupyter. The ROI always included a small part of the soma. For each stimulation condition, $\Delta \mathrm{F} / \mathrm{R}\left(\Delta \mathrm{F}_{\text {green }} / \mathrm{F}_{\text {red }}\right)$ values were calculated on a pixel-by-pixel basis. $\Delta \mathrm{F} / \mathrm{R}$ values were then averaged across the length of the dendrite, and the peak between 360 and $720 \mathrm{~ms}$ was taken as the peak $\Delta \mathrm{F} / \mathrm{R}$ value per stimulation condition per cell ( start of stimulation $=300 \mathrm{~ms}$ ). Given that our analysis was based on averaging over the length of the dendrite, we discarded dendrites $<35$ $\mu \mathrm{m}$. To quantify how $\Delta \mathrm{F} / \mathrm{R}$ signals varied along the dendrite in cells which displayed a calcium event, we averaged $\Delta \mathrm{F} / \mathrm{R}$ signals between 450 and $690 \mathrm{~ms}$ and plotted the $\Delta \mathrm{F} / \mathrm{R}$ over dendritic location for each repetition. These curves were box smoothed in Igor Pro, and the location (relative to the soma) of the peak calcium signal of all repetitions was averaged per cell. Software packages used for the data analysis included Python version 2.7.15, NumPy version 1.16.1, SciPy version 1.2.1, and Pandas version 0.18 .0. For the analysis of synaptically evoked calcium signals, we either searched for the largest calcium transient and extracted a 5-pixel-wide average over time (see Fig. 6) or calculated the integral of $\Delta \mathrm{F} / \mathrm{F}\left(\Delta \mathrm{F}_{\text {green }} / \mathrm{F}_{\text {green }}\right)$ signals (see Fig. 7; MATLAB, The MathWorks). For the latter, all $\Delta \mathrm{F} / \mathrm{F}$ values were summed over the length of the dendrite and duration of the response $(300 \mathrm{~ms})$. Since the total dendritic length often exceeded the imaging window, the ROI was traced to span the entire stretch of dendrite contained in the imaging window. Thus, the summation of $\Delta \mathrm{F} / \mathrm{F}$ values assumes a similar dendritic length across cells. 
The relative contribution of AMPARs and NMDARs was estimated pharmacologically.

Experimental design and statistical analysis. Electrophysiological recordings were performed in MSO cells from different brain slices of each animal. Statistical significance between normal acoustic environment (NAE) and OWN groups at a given age was tested with either an unpaired $t$ test or a Mann-Whitney $U$ test. To test whether a parameter changed significantly over the course of development within a given condition, either a one-way ANOVA or a Kruskal-Wallis test was used to control for multiple comparisons. For clarity, only the earliest developmental stage at which a statistically significant difference was found in relation to the latest developmental stage tested is reported in the text. Statistical analysis was performed in Prism 8 (version 8.1.1). The data plotted are the median and interquartile range, as most of the data were not normally distributed.

\section{Results}

Developmental decrease in the whole-cell calcium current and loss of a rapidly inactivating calcium current

Somatic whole-cell calcium currents were recorded in MSO neurons from P10 to P60 $(n=75)$ to assess their developmental regulation. Pharmacologically isolated calcium currents were elicited from a holding potential of $-85 \mathrm{mV}$ by a $400 \mathrm{~ms}$ voltage step ranging from $-70 \mathrm{mV}$ to $56 \mathrm{mV}$ incremented in $7 \mathrm{mV}$ steps (Fig. 1A,B). Two main differences were apparent between current-voltage relationship curves at P10 and P20. First, the peak of the whole-cell calcium current was larger in P10 neurons. Second, a low voltage-activated calcium current was evident at P10 (Fig. 1A, arrow). The shape of the current-voltage relationship and the more pronounced inactivation at P10 indicated the presence of T-type channels in juvenile, but not adult animals. Therefore, T-type currents were isolated with a subtraction protocol incremented in $5 \mathrm{mV}$ steps (Fig. 1C,D). In juvenile cells, the prehyperpolarization unmasked a rapidly inactivating inward current that was absent in adult animals (Fig. 1C,D).

Throughout development, the whole-cell calcium current increased slightly between P10 ( 2 nA. $n=9)$ and P13 (P11: $2.6 \mathrm{nA}$, $n=8 ; \mathrm{P} 13: 2.6 \mathrm{nA}, n=13)$, before progressively decreasing to mature levels (P14: $2.1 \mathrm{nA}, n=13 ; \mathrm{P} 15: 2.1 \mathrm{nA}, n=11 ; \mathrm{P} 16: 2 \mathrm{nA}$, $n=5$; P20: $1.4 \mathrm{nA}, n=9$; P60: $1.1 \mathrm{nA}, n=7 ; F_{(7,67)}=13.18, p<$ 0.0001 , one-way ANOVA; Dunnett's test, P10 vs P60, $p<0.0001$; Fig. $1 E$ ). In turn, a large T-type current measured at $-35 \mathrm{mV}$ at P10 (497 pA, $n=10$ ) decreased rapidly a few days after hearing onset to undetectable levels at P60 (P11: $371 \mathrm{pA}, n=10 ; \mathrm{P} 13: 115$ $\mathrm{pA}, n=17$; P14: $101 \mathrm{pA}, n=18$; P15: $53 \mathrm{pA}, n=13 ; \mathrm{P} 16: 36 \mathrm{pA}$, $n=7$; P20: $32 \mathrm{pA}, n=9$; P60: $32 \mathrm{pA}, n=7)(n=91 ; \mathrm{H}=62.90$, $p<0.0001$, Kruskal-Wallis; Dunn's test, P10 vs P60, $p<0.0001$; Fig. $1 F)$. To assess whether the developmental regulation of the whole-cell calcium current is affected by changes in acoustic experience, we repeated these experiments in gerbils raised in OWN (peak current, $n=43$; T-type, $n=50$ ). The whole-cell calcium current was significantly smaller at P13 ( $2 \mathrm{nA}$ in OWN, $n=12$ vs $2.6 \mathrm{nA}$ in an NAE, $p=0.005$; P14 OWN vs P14 NAE, $p=0.79$; P15 OWN vs P15 NAE, $p=0.09$, unpaired $t$ test; Fig. $1 E$, yellow symbols). Similarly, the T-type calcium current seemed to decrease faster, with a significantly smaller current amplitude at P14 (53.1 pA in OWN, $n=15$ vs $101.1 \mathrm{pA}$ in NAE, $p=0.03$; $\mathrm{P} 13$ OWN vs P13 NAE, $p=0.58$; P15 OWN vs P15 NAE, $p=0.28$, Mann-Whitney $U$; Fig. $1 F$, yellow symbols). Together, the peak somatic calcium current decreases from before hearing onset to maturity, and a T-type component is rapidly downregulated after hearing onset. Exposure to OWN appears to accelerate this developmental profile.
A
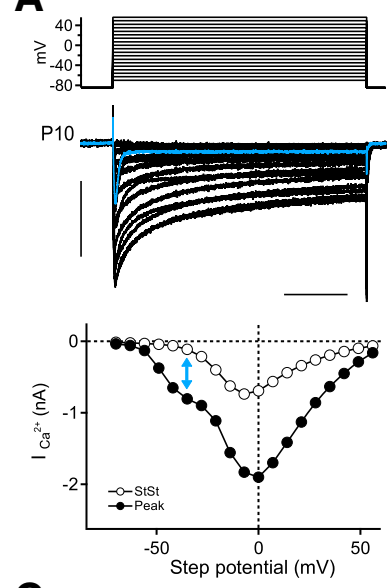

C

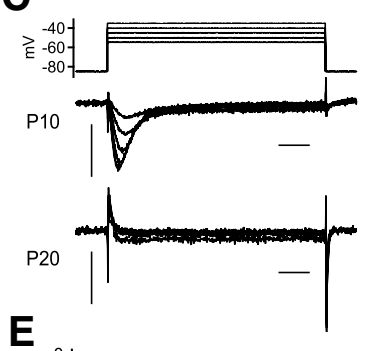

E 0



B

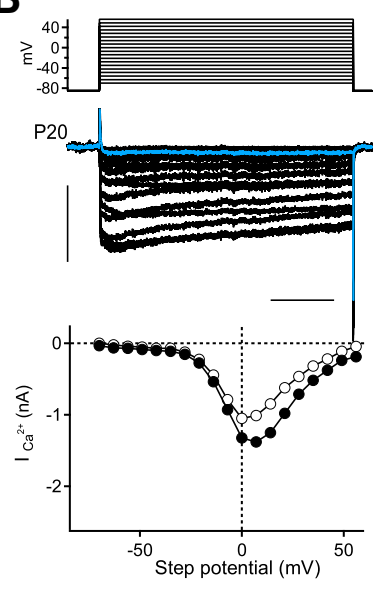

D


Figure 1. Developmental refinement of whole-cell calcium currents in MSO neurons. $\boldsymbol{A}, \mathrm{Top}$, Whole-cell calcium current in a P10 MS0 neuron. Calibration: 100 ms, 1 nA. Blue trace represents the whole-cell current at a step potential of $-35 \mathrm{mV}$. Bottom, Current-voltage relationship of the whole-cell calcium current. Blue arrow indicates the low voltage-activated T-type component at a step potential of $-35 \mathrm{mV}$. Step potential was increased in $7 \mathrm{mV}$ increments. Black circles represent peak current. Open circles represent steady-state current. Calcium currents were pharmacologically isolated with $1 \mu \mathrm{M}$ TTX, 2 mM 4-AP, 10 mm tetraethylammonium chloride, $50 \mu \mathrm{m}$ ZD 7288, $20 \mu \mathrm{m}$ DNQX, $50 \mu \mathrm{m}$ D-AP5 or $10 \mu \mathrm{m}$ R-CPP, $0.5 \mu \mathrm{m}$ strychnine, and 10 $\mu \mathrm{M}$ SR 95531 in the presence of $2.5 \mathrm{~mm} \mathrm{CaCl}_{2}$ and $0.5 \mathrm{~mm} \mathrm{MgCl}$. Calcium currents were $\mathrm{P} / \mathrm{x}$ corrected. $\boldsymbol{B}$, Same as in $\boldsymbol{A}$, but in a P20 MSO neuron. Calibration: $100 \mathrm{~ms}, 1 \mathrm{nA}$. C, Whole-cell calcium current evoked by a step command to $-55 \mathrm{mV}$ (incremented in $5 \mathrm{mV}$ steps) when preceded by a prepulse to $-85 \mathrm{mV}$ to remove steady-state inactivation in a P10 (top) and P20 (bottom) MSO neuron. Calibration: $10 \mathrm{~ms}, 500$ pA. D, Whole-cell calcium current evoked by a step command to $-55 \mathrm{mV}$ (incremented in $5 \mathrm{mV}$ steps) without a prepulse in a P10 (top) and P20 (bottom) MSO neuron. Calibration: 10 ms, 500 pA. $\boldsymbol{E}$, Change in the maximal peak wholecell calcium current extracted from the protocol in $\boldsymbol{A}$ during late postnatal development in gerbils raised in an NAE (black symbols) and gerbils raised in OWN (yellow symbols). Maximal currents were evoked by a step potential to $-7,0$, or $7 \mathrm{mV}$. Dashed line indicates hearing onset (P12). F, Development of the T-type calcium current at a step potential of $-35 \mathrm{mV}$, measured with a subtraction protocol during late postnatal development in gerbils raised in an NAE (black symbols) and in gerbils raised in OWN (yellow symbols). A sigmoid was fitted to the data. Sigmoid half value for NAE is at postnatal day 11.1. Dashed line indicates hearing onset (P12). Symbols represent the median. Error bars indicate the first and third quartiles. ${ }^{*} p<0.05$.

OWN exposure accelerates the loss of action potential-evoked dendritic calcium transients during postnatal development

As the size of action potentials (Scott et al., 2005; Chirila et al., 2007; Winters and Golding, 2018) and the whole-cell calcium currents of MSO neurons are developmentally regulated, calcium signals driven by action potentials are likely to change during development. To test this hypothesis, we imaged dendritic cal- 
A
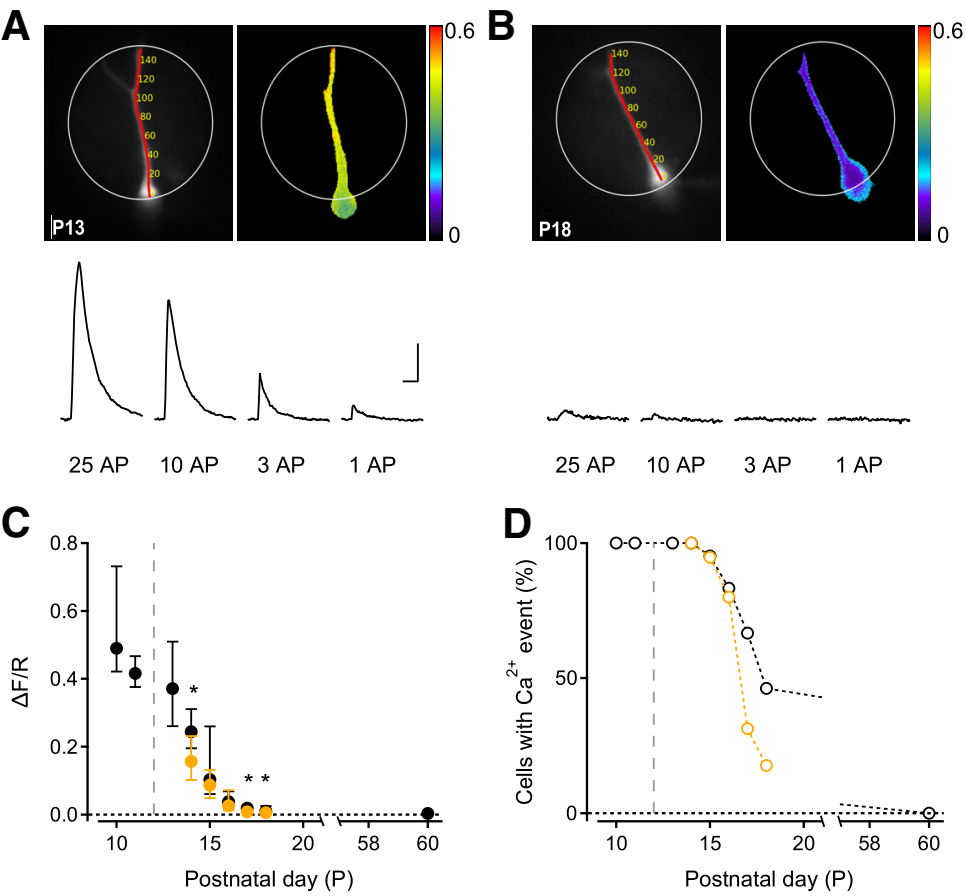

D


Figure 2. Developmental downregulation of dendritic calcium influx triggered by action potentials is accelerated by noise rearing. $\boldsymbol{A}$, Dendritic calcium transient induced by a train of 25 somatically evoked action potentials at $10 \%$ above current threshold in a P13 MSO neuron. Top, Dendrite filled with OGB-1 as visualized during recordings (left) and color mapped for $\Delta \mathrm{F}_{\text {green }} / \mathrm{F}_{\text {red }}$ (right). White circle represents the location of the circular field stop. Bottom, Dendritic calcium transients $\left(\Delta \mathrm{F}_{\text {green }} /\right.$ $\left.\mathrm{F}_{\text {red }}\right)$ in response to trains of 25,10 , and 3 action potentials $(100 \mathrm{~Hz})$ and in response to 1 action potential in the same $P 13$ neuron. The calcium signal was averaged over the length of the visible dendrite. Calibration: $500 \mathrm{~ms}, 0.1 \Delta \mathrm{F}_{\text {green }} / \mathrm{F}_{\text {red }}$. $\boldsymbol{B}$, Same as in $\boldsymbol{A}$, but in a P18 neuron. A detectable calcium transient could only be evoked in response to a train of 10 and 25 action potentials. $\boldsymbol{C}$, Development of the dendritic calcium influx $\left(\Delta \mathrm{F}_{\text {green }} / \mathrm{F}_{\text {red }}\right)$ evoked by 25 action potentials at $10 \%$ above the current threshold in gerbils raised in an NAE (black symbols) and in gerbils raised in OWN (yellow symbols). Dashed line indicates hearing onset (P12). D, Percentage of cells that display a dendritic calcium event in response to 25 action potentials throughout late postnatal development. Black symbols represent NAE. Yellow symbols represent OWN. Dashed line indicates hearing onset (P12). E, Amplitude of dendritic calcium transients $\left(\Delta \mathrm{F}_{\text {green }} / \mathrm{F}_{\text {red }}\right)$ along the dendrite of a P11 (top, pink) and P15 (bottom, blue) neuron (NAE) in response to 25 action potentials. In both cases, dendritic location " $0 \mu \mathrm{m}^{\prime \prime}$ represents the soma. Dashed arrows indicate the dendritic location corresponding to the maximal $\Delta F_{\text {green }} / F_{\text {red }}$ value. $F$, Developmental change in the dendritic location of the maximum $\Delta \mathrm{F}_{\text {green }} / \mathrm{F}_{\text {red }}$ value in response to 25 action potentials in P10-P16 cells that displayed a calcium event. Dendritic location " $0 \mu \mathrm{m}$ " indicates the soma. Dashed lines indicate the median dendritic lengths of the dataset. Black symbols represent NAE. Yellow symbols represent OWN. Filled symbols represent the median. Error bars indicate the first and third quartiles. ${ }^{*} p<0.05$.

cium signals evoked by somatic action potentials. Dendritic calcium responses were measured in response to a single action potential, and trains of 3,10 , and 25 action potentials at $100 \mathrm{~Hz}$. A large global calcium influx could be reliably evoked in MSO neurons at P13 $\left(\Delta \mathrm{F}_{\text {green }} / \mathrm{F}_{\text {red }}\right.$ for 25 APs: $0.37, n=13$; Fig. $\left.2 A\right)$. Even single action potentials were sufficient to evoke a global dendritic calcium signal in all P10 and P11 cells, and in almost all P13 cells. However, by P18, only a fraction of cells displayed a small calcium signal and only in response to 10 or 25 action potentials $\left(\Delta \mathrm{F}_{\text {green }} / \mathrm{F}_{\text {red }}\right.$ for 25 APs: $0.01, n=13$; Fig. $\left.2 B\right)$. To determine the developmental profile of global dendritic calcium signals driven by somatic action potentials, we performed these experiments between P10 and P60 $(n=124)$. Given that calcium signals were only observed in response to 25 action potentials at $\mathrm{P} 18$, we restricted the analysis to this stimulation condition. Before hearing onset, calcium signals were even larger than at $\mathrm{P} 13\left(\Delta \mathrm{F}_{\text {green }} / \mathrm{F}_{\text {red }}\right.$ for 25 APs at P10: $0.49, n=11 ; \mathrm{P} 11: 0.42, n=$ 14). Briefly after hearing onset, the dendritic calcium transients declined rapidly $\left(\Delta \mathrm{F}_{\text {green }}\right)$ $\mathrm{F}_{\text {red }}$ for 25 APs at P14: 0.24, $n=19$; P15: $0.10, n=21 ; \mathrm{P} 16: 0.04, n=12 ; \mathrm{P} 17: 0.02$, $n=12$; P18: 0.01, $n=13$; Fig. $2 C$ ). In neurons from P60 animals, no calcium transient could be elicited in the dendrite (P60: $0, n=9$; Fig. $2 C$; $\mathrm{H}=90.1, p<$ 0.0001, Kruskal-Wallis; Dunn's test, P10 vs P60, $p<0.0001)$. Raising gerbils in OWN $(n=88)$ led to a slight but significant decrease in the amplitude of calcium transients at P14, P17, and P18 $\left(\Delta \mathrm{F}_{\text {green }} /\right.$ $\mathrm{F}_{\text {red }}$ for $25 \mathrm{APs}$ at P14 NAE: 0.24 vs OWN: $0.16, n=16, p=0.02$; P17 NAE: 0.02 vs OWN: $0.008, n=16, p=0.01$; P18 NAE: 0.01 vs OWN: $0.005, n=17, p=0.01$, Mann-Whitney $U$ but not at P15, $p=$ 0.15 , and P16, $p=0.47$; Fig. $2 C$, yellow symbols), indicating an accelerated loss of dendritic calcium signals. In more detail, we found that the frequency of calcium events differed between the NAE and OWN conditions. While a calcium transient could always be evoked in response to 25 action potentials between P10 and P14, only $46.2 \%$ of cells displayed a calcium transient at $\mathrm{P} 18$ in animals raised in an NAE (Fig. 2D). The occurrence of calcium transients appeared to decline more rapidly in gerbils exposed to OWN, with only $31.3 \%$ and $17.6 \%$ of cells displaying a calcium event in response to 25 action potentials at $\mathrm{P} 17$ and P18, respectively (compared with $66.7 \%$ and $46.2 \%$ in gerbils raised in an NAE; Fig. 2D, yellow symbols). Together, OWN accelerates the developmental refinement of action potential-evoked dendritic calcium transients.

Furthermore, the spatial profile of calcium transients along the imaged dendrite appeared to change during late postnatal development $(n=84)$. Until shortly after hearing onset, similar to neurons of the lateral superior olive (Kullmann and Kandler, 2008), the largest calcium responses were observed at distal locations (P10: $46.9 \mu \mathrm{m}, n=$ 11; P11: $53.5 \mu \mathrm{m}, n=14$; P13: $63.6 \mu \mathrm{m}, n=13$; P14: $50.5 \mu \mathrm{m}$, $n=19)$. After P14, however, calcium signals tended to peak at more proximal locations (P15: $28.7 \mu \mathrm{m}, n=18$; P16: $25.7 \mu \mathrm{m}$, $n=9 ; \mathrm{H}=20.74, p=0.0009$, Kruskal-Wallis; Dunn's test, P11 vs P16, $p=0.02$; Fig. $2 E, F)$. After P16, the further developmental reduction in the size of calcium transients prevented the quanti- 
fication of their location. While Figure $2 E$ shows an example of a $\mathrm{P} 15$ cell with a larger $\Delta \mathrm{F}_{\text {green }} / \mathrm{F}_{\text {red }}$ in proximal regions, many P15 and P16 neurons displayed calcium signals, which varied only little along the dendrite. Thus, the developmental change in peak $\Delta \mathrm{F}_{\text {green }} / \mathrm{F}_{\text {red }}$ may reflect a loss of distal calcium signals, which leads to an apparent shift in calcium entry sites. Our finding that larger calcium transients were located distally before P15 did not simply result from an age-dependent difference in the dendritic length of neurons (Fig. 2F, dotted lines). Finally, raising gerbils in OWN $(n=49)$ did not result in significant changes in the location of the peak $\Delta \mathrm{F}_{\text {green }} / \mathrm{F}_{\text {red }}$ signal along the dendrite (P14: 32.5 $\mu \mathrm{m}, n=16, p=0.35$; P15: $28 \mu \mathrm{m}, n=18, p=0.55$; P16: 22.9 $\mu \mathrm{m}, n=15, p=0.94$, Mann-Whitney $U$; Fig. $2 F)$. In summary, large global calcium transients, likely through VGCCs, can be evoked by somatic action potentials in MSO dendrites, and their developmental loss is accelerated by OWN.

\section{Membrane properties remain unaltered by $\mathrm{OWN}$ rearing}

Since rearing animals in OWN modified the calcium currents and action potential-evoked dendritic calcium transients, we investigated whether the developmental profile of voltage signaling is also affected. As described previously (Scott et al., 2005; Chirila et al., 2007; Winters and Golding, 2018), the shape of the action potential changed substantially over the course of late postnatal development (Fig. 3A). The current threshold in response to a short ramp stimulation decreased between P10 (6.2 nA, $n=18)$ and P14 ( $3.5 \mathrm{nA}, n=29)$, before increasing again to mature levels (5.6 nA, $n=10$; overall NAE, $n=170$; OWN, $n=103$ ). The repolarizing phase of the action potential was strongly developmentally regulated (NAE, $n=133$; OWN, $n=93$ ). At P10, action potentials almost exclusively displayed a depolarizing afterpotential as large as $11 \mathrm{mV}(\mathrm{P} 10: 4.9 \mathrm{mV}, n=12)$. Only a day later, 8 of 15 cells displayed a depolarizing after-potential, whereas 7 of 15 cells developed a small and prolonged afterhyperpolarization (P11: $1.2 \mathrm{mV}, n=15)$. From P13 onward, all cells displayed an after-hyperpolarization that progressively became larger and faster before reaching mature levels (P60: -5 $\mathrm{mV}, n=9)\left(F_{(8,124)}=68.03, p<0.0001\right.$, one-way ANOVA; Dunnett's test, P10 vs P60, $p<0.0001$; Fig. 3B). Alongside these changes, the size of somatic action potentials decreased gradually from P10 $(83 \mathrm{mV}, n=12)$ to $\mathrm{P} 60(23.6 \mathrm{mV}, n=9)$ (NAE, $n=$ 133; OWN, $n=93$; $\mathrm{H}=91.29, p<0.0001$, Kruskal-Wallis; Dunn's test, P10 vs P60, $p<0.0001$; Fig. $3 C$ ). Finally, both the input resistance and the membrane time constant (NAE, $n=$ $151 / 150$; OWN, $n=118$ ) decreased substantially from P10 (112 $\mathrm{M} \Omega ; 8.1 \mathrm{~ms}, n=12)$ to $\mathrm{P} 60(4.8 \mathrm{~m} \Omega ; 436 \mu \mathrm{s}, n=13)\left(\mathrm{R}_{\mathrm{in}}: \mathrm{H}=\right.$ $132.3, p<0.0001$, Kruskal-Wallis, Dunn's test, P10 vs P60, $p<$ 0.0001 ; Tau onset $: \mathrm{H}=119.4, p<0.0001$, Kruskal-Wallis, Dunn's test, P10 vs P60, $p<0.0001$; Fig. $3 D, E)$. Importantly, raising gerbils in OWN did not induce substantial changes in the parameters tested. Together, the changes in somatic passive and active membrane properties during late postnatal development remain unaffected by exposure to OWN. Thus, we speculate that the loss of dendritic calcium transients is at least partially driven by a change in the amount or properties of VGCCs.

\section{Refinement of excitatory inputs to MSO neurons}

Calcium-permeable glutamate receptors also enable calcium influx in neurons. To obtain an initial view of the refinement of this source of calcium influx, we studied the developmental redistribution of presumably glutamatergic axonal inputs to MSO nuclei. Here, we took advantage of the fact that different calcium binding proteins are distinctly distributed in glycinergic and glu-
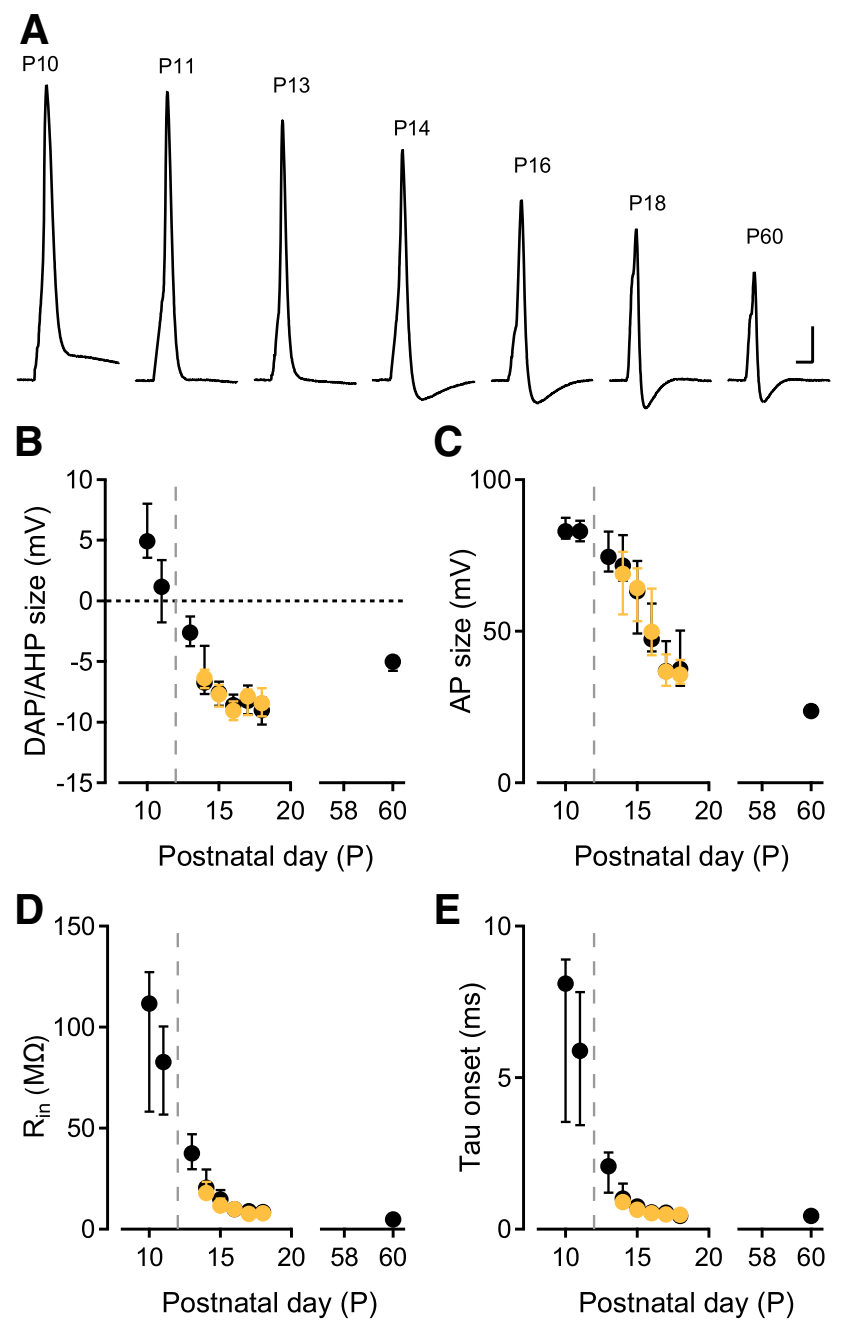

Figure 3. Noise rearing does not affect the development of action potential and resting membrane parameters. $\boldsymbol{A}$, Shape of the action potential at $10 \%$ above the current threshold throughout late postnatal development (P10-P18) and at maturity (P60). Calibration: 1 ms, 10 $\mathrm{mV}$. $\boldsymbol{B}$, Development of the depolarizing after potential (DAP) or after-hyperpolarizing potential (AHP). C, Developmental change in the action potential size (from baseline). $\boldsymbol{D}, \boldsymbol{E}$, Change in the input resistance (Rin) and membrane time constant as a function of postnatal day. All symbols represent the median. Error bars indicate the first and third quartiles. $\boldsymbol{B}-\boldsymbol{E}$, Black symbols represent NAE. Yellow symbols represent OWN. Gray dashed line indicates hearing onset (P12).

tamatergic inputs to MSO neurons (Couchman et al., 2010). Calbindin is known to label glycinergic inputs and was found to target the soma of MSO neurons from P14 onwards (Fig. 4A), corroborating earlier findings (Kapfer et al., 2002). Calretinin, which marks glutamatergic inputs to MSO neurons, labeled most strongly in the neuropil surrounding the MSO soma in P9 animals (Fig. 4B). As the animals matured, the staining revealed more and larger synaptic-like structures on thick dendritic trunks (Fig. 4B). This indicates that the structure of excitatory synapses refines during late postnatal development and might hint at an accompanying change in synaptic physiology. To conclude the development of calcium binding proteins, we performed parvalbumin stainings (Fig. 4C). As in other mammals (Caicedo et al., 1996; Lohmann and Friauf, 1996), parvalbumin expression started around hearing onset and was the only major calcium binding protein expressed postsynaptically. Thus, calcium buffering will also be developmentally regulated. Finally, by detecting vesicular glutamate transporter 1 (Vglut1), the developmental 


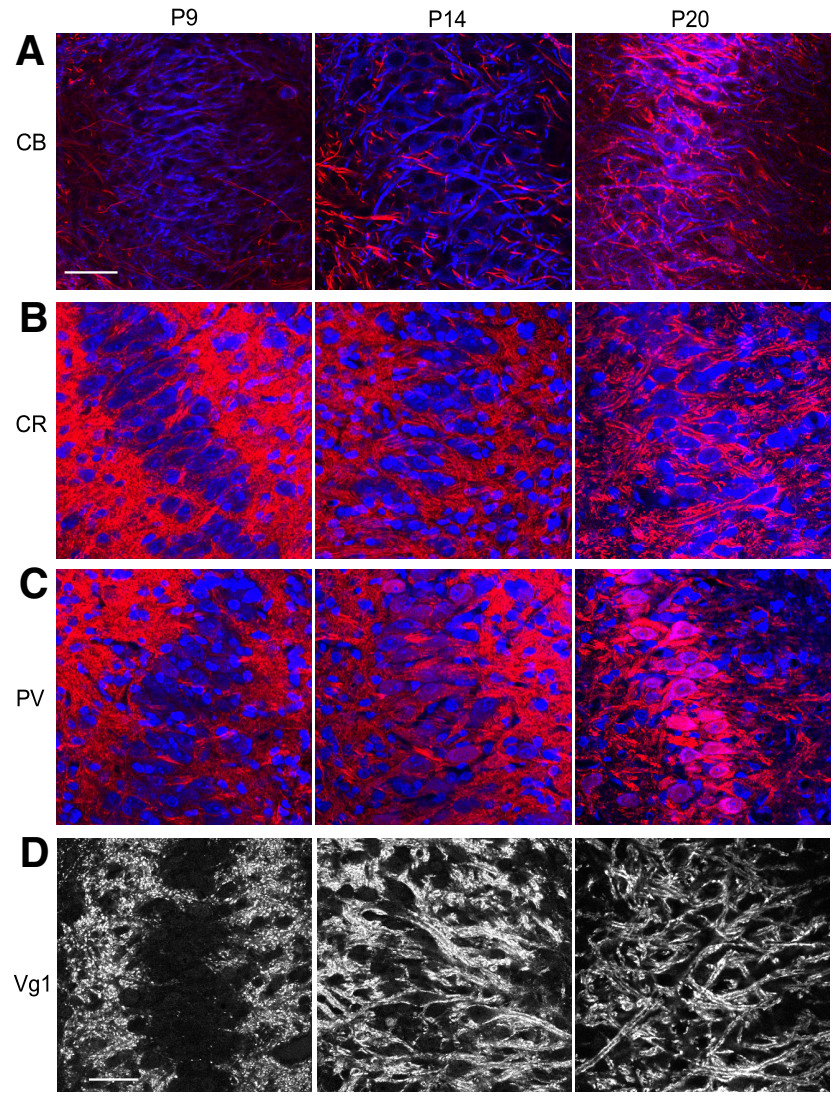

Figure 4. Synaptic marker proteins reveal large morphological rearrangements during synaptic development. $\boldsymbol{A}$, Calbindin (red) and MAP-2 (blue) labeling of P9, P14, and P20 MSO sections shows the rearrangement of presumably glycinergic inputs. Scale bar, $40 \mu \mathrm{m} . \boldsymbol{B}$, Calretinin (red) and Nissl (blue) labeling of P9, P14, and P20 MSO sections shows the rearrangement of presumably glutamatergic inputs. Scaled as in A. C, Parvalbumin (red) and Nissl (blue) labeling of P9, P14, and P20 MSO sections shows the increase in postsynaptic expression of calcium buffer. Scaled as in $\boldsymbol{A}$. D, Vesicular glutamate transporter 1 (here abbreviated as Vg1) labeling shows the rearrangement of glutamatergic inputs to MSO neurons during late postnatal development at P9, P14, and P20. Scale bar, $40 \mu \mathrm{m}$.

refinement from small glutamatergic synaptic structures to large structures surrounding MSO dendrites was demonstrated (Fig. $4 D$ ) and corroborates the assumption that calretinin labels excitatory inputs.

\section{Development of excitatory synaptic transmission in MSO neurons}

Since glutamatergic inputs display a structural refinement, we examined the late postnatal development of glutamatergic synaptic transmission. We used minimal fiber stimulation to characterize developmental changes in the size and kinetics of the single-fiber AMPAR-mediated EPSCs $(n=51)$. From P11 to P17, the EPSC size increased significantly (P11: $-1.07 \mathrm{nA}, n=20$; P14: $-1.54 \mathrm{nA}, n=17$; P17: $-1.89 \mathrm{nA}, n=14 ; \mathrm{H}=15.09, p=$ 0.0005 , Kruskal-Wallis Dunn's test for P11 vs P17, $p=0.003$; Fig. $5 A, B$, left) and the decay time constant decreased significantly (P11: $396 \mu s ;$ P14: $297 \mu s ; \mathrm{P} 17: 266 \mu s ; \mathrm{H}=11.54, p=0.003$, Kruskal-Wallis, Dunn's test, P11 vs P17, $p=0.003$; Fig. 5A, $C$, left). In OWN-raised animals $(n=16)$, the size of single-fiber AMPAR-mediated EPSCs was considerably, but not significantly, larger at $\mathrm{P} 14(-2.34 \mathrm{nA}, n=7$ in OWN vs $-1.54 \mathrm{nA}$ in NAE, $p=$ 0.1 , Mann-Whitney $U)$ and exhibited no change at P17 $(-1.59$ $\mathrm{nA}, n=9$ in OWN vs $-1.89 \mathrm{nA}$ in NAE, $p=0.9$; Fig. $5 B$, right). OWN exposure significantly decreased the EPSC decay time con-
A




G

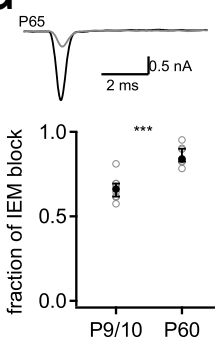



H
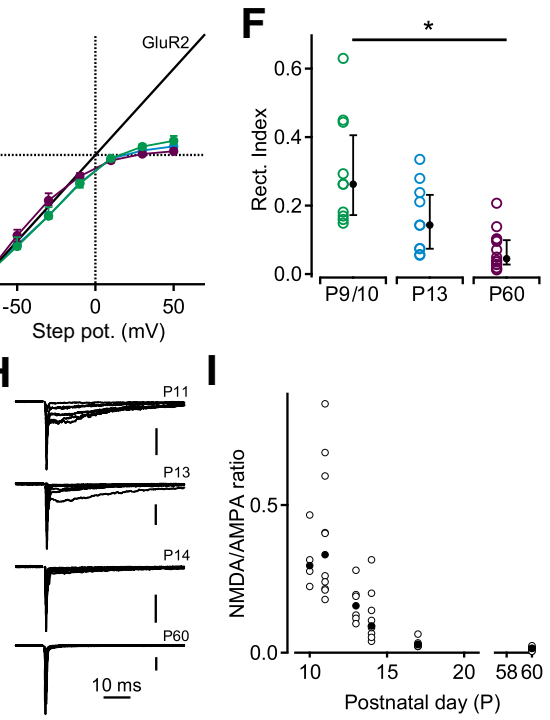

Figure 5. Development of excitatory inputs to MSO neurons is slightly accelerated by noise rearing. $\boldsymbol{A}$, Left, Synaptic response to a minimal fiber stimulation protocol in an MSO neuron at P11, P14, and P17. Right, Overlay of the normalized synaptic responses in left image, highlighting the difference in decay kinetics. $\boldsymbol{B}$, Single-fiber EPSC size during late postnatal development in gerbils raised in an NAE (left) and in OWN (right). $C$, The decay kinetics of the single-fiber EPSC during late postnatal development in gerbils raised in an NAE (left) and in OWN (right). ${ }^{*} p<$ 0.05. D, Example traces of the current-voltage relationship of the AMPAR-mediated EPSC at P9 (top) and P60 (bottom) normalized to the current recorded at the most negative step potential. $\boldsymbol{E}$, Normalized current-voltage relationship of AMPAR currents. Black line indicates the expected response for GluR2 only AMPARs. $\boldsymbol{F}$, RI of the AMPAR-mediated EPSC at P9/10, P13, and $P 60$. The RI was calculated by dividing the peak EPSC at $50 \mathrm{mV}$ with the corresponding value of a line fitted to the first four values ( $\boldsymbol{E}$, black line). Filled symbols represent the median. Error bars indicate the first and third quartiles. ${ }^{*} p<0.05$. G, Single-fiber EPSC (top) before (black) and after (gray) application of $60 \mu \mathrm{m}$ IEM-1460. Bottom, The fraction of EPSC block between control and drug conditions. Open symbols represent individual cells. Closed symbols represent the median with quartiles. ${ }^{* * *} p<0.001$. $\boldsymbol{H}$, Synaptic currents of multiple inputs recorded in $<0$ $\mathrm{Mg}^{2+}$ external concentration at different developmental stages. In adult animals, the slow inward, presumably NMDAR-mediated current is absent. Calibration: 2 nA.I, NMDA/AMPA ratio extracted from currents exemplified in $\boldsymbol{H}$ as a function of postnatal day.

stant at P14 (227 $\mu$ s in OWN vs $297 \mu$ s in NAE, $p=0.01$, MannWhitney $U$ ) but not at P17 (262 $\mu$ s in OWN vs $266 \mu$ s in NAE, $p=0.6$; Fig. $5 C$, right).

The decay kinetics of synaptically evoked EPSCs are partly determined by the subunit composition of synaptic receptors. In particular, the presence of the GluR2 subunit of AMPARs has been shown to correlate negatively with fast channel gating (Geiger et al., 1995). The presence of the GluR2 subunit reduces the calcium permeability (Hollmann et al., 1991; Burnashev et al., 1992) and the rectification of AMPARs mediated by endogenous intracellular polyamines (Hollmann et al., 1991; Bowie and Mayer, 1995; Kamboj et al., 1995; Koh et al., 1995). Since the 
synaptic decay kinetics were developmentally regulated, we evaluated the rectification of synaptic AMPARs to gain insight into their subunit composition by recording current-voltage relationships with $100 \mu \mathrm{M}$ spermine added to the pipette $(n=34$; Fig. $5 D-F)$. AMPAR-mediated currents exhibited significantly larger rectification at P60 compared with P9/10 (RI P9/10: 0.26, $n=10$; P13: 0.14, $n=10$; P60: 0.04, $n=14 ; \mathrm{H}=18.43, p<0.0001$, Kruskal-Wallis, Dunn's test, P9-P10 vs P60, $p<0.0001$; Fig. $5 D-F)$, indicating a developmental reduction of GluR2 subunits. Pharmacologically, the lack of GluR2 subunits in AMPARs can be demonstrated by their susceptibility to the open channel blocker IEM-1460. In P9 animals, the size of EPSCs evoked by a single fiber was blocked by $66 \%(n=8$; control: -1.13 nA; IEM-1460: $-0.39 \mathrm{nA}$; Wilcoxon test, $p=0.0078)$. In P9/10 animals, IEM1460 blocked the EPSCs by $84 \%(n=7$; Fig. $5 G)$. This significant reduction (Wilcoxon test, $p=0.0156$ ) was on average based on a current reduction from -1.86 to $-0.24 \mathrm{nA}$ (Fig. $5 G$ ). As for the rectification, the IEM-1460 experiment demonstrates that the fraction of calcium-permeable AMPARs increases significantly between P9 and P60 (Mann-Whitney $U$ test, $p=0.0006$ ). Thus, as indicated by the developmental speeding and an increase in the strong rectification of the AMPAR-mediated EPSC, mature MSO neurons predominantly express calcium-permeable AMPARs most likely composed of GluR4 subunits.

The developmental reduction in GluR2 subunits is indicative of increased calcium permeability through AMPARs. However, the major source of synaptic calcium influx is commonly the NMDAR. Therefore, we also determined the developmental profile of NMDAR signaling by recording NMDA/AMPA current ratios. We stimulated afferent fibers in the absence of extracellular $\mathrm{Mg}^{2+}$ to allow ions to permeate through NMDARs held at $-60 \mathrm{mV}$ (Fig. 5H). The peak current was considered as the AMPAR component, and the current size $5 \mathrm{~ms}$ after the peak was taken as the NMDAR component. Corroborating data from rat MSO neurons (Smith et al., 2000), our data show a strong downregulation of NMDARs during late postnatal development $(n=$ $45)$, indicated by the drop in NMDA/AMPA ratio of 0.295 at P10 $(n=4)$ to 0.014 at a P60 $(n=9)(\mathrm{H}=37.57, p<0.0001$, Kruskal-Wallis, Dunn's test, P10 vs P60, $p=0.0008$; Fig. $5 I$ ). This downregulation is extensive and leads to the loss of detectable NMDAR-mediated currents at P60.

Since NMDARs and the AMPAR GluR2 subunit appear developmentally downregulated, we tested whether excitatory inputs trigger calcium influx in juvenile and adult animals under physiological conditions of 1.2 and $1 \mathrm{~mm}$ extracellular $\mathrm{Ca}^{2+}$ and $\mathrm{Mg}^{2+}$, respectively. At $\mathrm{P} 12 / 13(n=8)$, the EPSPs in response to a stimulation of 25 pulses at $100 \mathrm{~Hz}$ summed slightly (Fig. 6A) and a local dendritic calcium transient could be observed (Fig. 6B) in response to 10 and 25 pulse trains ( 10 pulses: $0.074 \Delta \mathrm{F}_{\text {green }} / \mathrm{F}_{\text {green }}$, 25 pulses: $0.142 \Delta \mathrm{F}_{\text {green }} / \mathrm{F}_{\text {green }}, p=0.0078$, Wilcoxon test; Fig. $6 C)$. Repeating the same experiments in animals older than P60, we found very brief nonsummating EPSPs (Fig. 6D). Again, local calcium transients dependent on the stimulation pulse number could be observed at distinct dendritic locations $(n=5,10$ pulses: $0.048 \Delta \mathrm{F}_{\text {green }} / \mathrm{F}_{\text {green }}, 25$ pulses: $0.077 \Delta \mathrm{F}_{\text {green }} / \mathrm{F}_{\text {green }}, p=0.0313$, Wilcoxon test; Fig. $6 E, F)$. Comparing the observed calcium transients between the different age groups ( 10 pulses: $p=0.171 ; 25$ pulses: $p=0.0186$; Mann-Whitney $U$ ) indicated a reduced calcium accumulation during 25 pulse trains in mature MSO neurons. Thus, as no NMDAR current was observed at P60 (Fig. $5 H, I)$, we propose that calcium transients in $>$ P60 were mediated largely by calcium-permeable AMPARs. Moreover, the con-


Figure 6. Synaptic inputs evoke calcium transients in juvenile and mature MSO dendrites under physiological conditions. A-C, Left, OGB-1-loaded dendrite of a P12 MSO neuron. Right, EPSPs evoked by train stimulations of afferent fibers. Left, Red circle represents the region from where the calcium transients in $\boldsymbol{B}$ were taken. $\boldsymbol{B}$, Local dendritic calcium transients in response to a 25-pulse stimulation (black trace) and a 10-pulse stimulation (gray trace). $C$, The maximal $\Delta \mathrm{F}_{\text {green }} / \mathrm{F}_{\text {green }}$ values from 8 dendrites (black lines and circles) and in addition the background signal (gray dashed line and circles). $\boldsymbol{D}-\boldsymbol{F}$, Same as in $\boldsymbol{A}-\boldsymbol{C}$, but for 5 dendrites from gerbils older than P60. D, Right, Gray inset, Magnified, first EPSP in the stimulation train.

tribution of calcium influx by locally activated VGCCs is likely minor, as calcium currents are substantially downregulated at that stage.

\section{Omnidirectional noise rearing accelerates the developmental} reduction of synaptically evoked dendritic calcium transients Next, we assayed the developmental profile of synaptically evoked calcium influx and the contribution of NMDARs and AMPARs in more detail under voltage-clamp conditions. To do so, we recorded synaptically evoked calcium responses in $0 \mathrm{~mm}$ $\mathrm{Mg}^{2+}$ extracellular solution. Synaptic stimulation evoked local dendritic calcium transients (Fig. $7 A, B$ ), which decreased in amplitude throughout development (P10: 230.1, $n=4$; P11: 367.4, $n=10 ; \mathrm{P} 13: 371.7, n=6$; P14: 244.1, $n=9 ; \mathrm{P} 17: 111.1, n=8$; P60: $33.5, n=11$ sum $\Delta \mathrm{F} / \mathrm{F} ; \mathrm{H}=26.46, p<0.0001$, KruskalWallis, Dunn's test, P11 vs P60 $p<0.0001$ ) (Fig. 7C). Importantly, a synaptic calcium response could still be evoked in P60, corroborating functional data from Figure 6. Following OWN exposure, synaptically evoked calcium transients at P14 were significantly smaller compared with NAE rearing (P14 OWN: 78.59, $n=6, p=0.026$; P17 OWN: 51.22, $n=9, p=0.42$, MannWhitney $U$ ) (Fig. 7C). Compared with action potential-evoked calcium transients, those evoked by synaptic stimulation, while local, did not appear to become spatially restricted during development.

In a subset of neurons $(n=35)$, we recorded synaptically evoked dendritic calcium transients before and after blocking AMPARs with DNQX. This procedure estimates the contribution of NMDARs and AMPARs to the overall calcium transient (Fig. 7D). At P11, the calcium influx was $72 \%$ mediated by NMDARs $(n=7)$, which peaked at P13 $(81 \%, n=5)$ before declining during development to $67 \%$ in P14 $(n=8)$ and $28 \%$ at P17 $(n=5)$. In adult animals, nearly no NMDAR contribution was observed $(n=10)$, consistent with our electrophysiological data $(\mathrm{H}=23.79, p<0.0001$, Kruskal-Wallis, Dunn's test, $\mathrm{P} 11 \mathrm{vs}$ $\mathrm{P} 60, p=0.0004)$. Rearing animals in OWN accelerated the developmental loss of the NMDAR contribution at P17 (Fig. 7D). In P14 OWN-reared animals, only $29 \%(n=5)$ of the synaptically evoked calcium transient was triggered by NMDARs (compared with $67 \%$ in NAE, $p=0.44$, Mann-Whitney $U$ ), whereas almost no NMDAR component was observed at P17 $(2.6 \%, n=6$ vs 
A
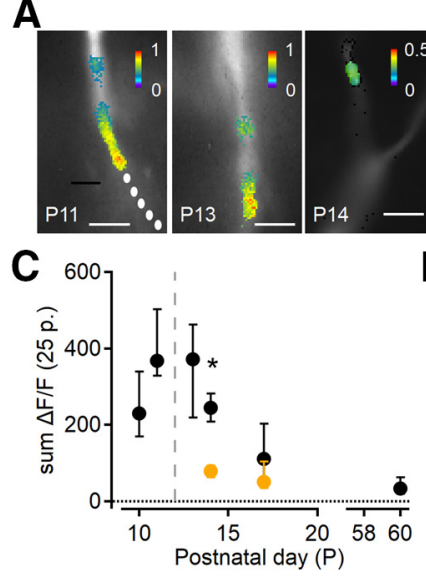

B

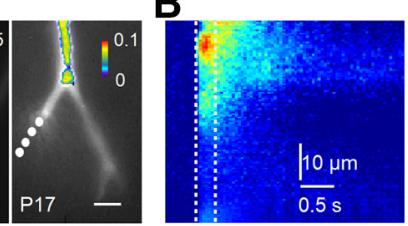

D 1 AMPANAE $=$ AMPAOWN



Figure 7. NMDAR contribution to synaptically evoked calcium transients in NAE- and OWN-reared animals. $\boldsymbol{A}$, Examples of dendritic calcium transients evoked by synaptic stimulation at several postnatal stages. The $R 01$ is colored for $\Delta F / F\left(\Delta F_{\text {green }} / F_{\text {green }}\right)$. White points indicate the out-of-focus part of the dendrite. Scale bar, $10 \mu \mathrm{m}$. $\boldsymbol{B}$, Example kymograph in a P14 neuron (shown in $\boldsymbol{A}$ ) illustrating synaptically evoked calcium influx over time along the imaged dendritic ROl. Dotted white lines indicate the region from which the calcium signal was summed. C, Overall decrease in the sum $\Delta \mathrm{F} / \mathrm{F}$ evoked by synaptic stimulation ( 25 pulses at $100 \mathrm{~Hz}$ ) in gerbils raised in an NAE (black symbols) and in gerbils raised in OWN (yellow symbols). Dashed line indicates hearing onset (P12). Filled symbols represent the median. Error bars indicate the first and third quartiles. ${ }^{*} p<0.05$. $\boldsymbol{D}$, The relative contribution of AMPARs and NMDARs toward the overall calcium influx during postnatal development assessed with pharmacology. Blue bars represent NAE. Yellow bars represent OWN. Open circles represent the NMDAR contribution (\%) to the calcium influx of individual cells, estimated by bath application of DNQX.

$28 \%$ in NAE, $p=0.03$, Mann-Whitney $U$ ). Together, synaptically evoked calcium transients decrease during late postnatal development to a small residual influx mediated by calciumpermeable AMPARs and OWN exposure accelerates this developmental decrease likely through a more rapid loss of NMDARs.

\section{Discussion}

Here we demonstrate that calcium signaling in MSO neurons is strongly downregulated during late postnatal development and that sound-driven activity accelerates this process. Large global calcium transients evoked by action potentials disappear shortly after hearing onset. Local synaptic calcium influx decreases during this developmental period but persists into adulthood. Thus, we describe a short time window of calcium signaling following hearing onset for developmental plasticity driven by specific experience-dependent cues.

\section{Developmental refinement of calcium currents and their activity dependence}

In immature MSO neurons, inactivating and noninactivating calcium currents are present and both are developmentally downregulated. While T-type-like currents fully disappear shortly after hearing onset, the noninactivating current decreases but persists into adulthood. The presence of somatic T-type calcium channels is in agreement with other auditory brainstem neurons (Doughty et al., 1998; Harasztosi et al., 1999).

As in other neurons, the presence of T-type calcium currents early in development might support neuronal growth and differentiation (Gu and Spitzer, 1993; Chambard et al., 1999; Autret et al., 2005; Lory et al., 2006; Levic et al., 2007). Moreover, given that T-type calcium channels require a prior hyperpolarization to relieve steady-state inactivation (Cueni et al., 2009), they may allow

inhibitory responses to influence calcium signals in developing MSO neurons. In support of such an interaction, medial nucleus of the trapezoid body-evoked hyperpolarizations in the lateral superior olive of neonatal gerbils elicit rebound action potentials, an effect that was speculated to involve low-threshold calcium currents (Sanes, 1993), and to be the cellular basis for the activitydependent refinement of medial nucleus of the trapezoid body arbors (Sanes and Takács, 1993).

The dendritic calcium transients induced by somatic action potentials are presumably driven by the activation of VGCCs through back-propagating action potentials. One explanation for the developmental loss of global, dendritic calcium transients is that downregulated back-propagating action potentials (Winters and Golding, 2018) will lose the ability to gate VGCCs efficiently at distal dendritic locations. Additionally, the slightly faster decrease of T-type and noninactivating calcium currents upon OWN may add to the different developmental profile of dendritic calcium signals in the distinct rearing conditions. Thus, the amount or distribution of calcium channels together with the reduction in action potential size likely defines the developmental loss of dendritic, global dendritic calcium transients. Therefore, mature MSO neurons are unusual in that their postsynaptic activity is not correlated with calcium influx.

\section{Developmental refinement of excitatory inputs and synaptically elicited calcium transients}

In auditory brainstem circuits, it is well established that glutamatergic synapses refine during postnatal development through a speeding of, and an increase in, AMPAR- (Bellingham et al., 1998; Taschenberger and von Gersdorff, 2000; Futai et al., 2001; Iwasaki and Takahashi, 2001; Joshi and Wang, 2002; Youssoufian et al., 2005; Case et al., 2011; Pilati et al., 2016) and a decrease in NMDAR-mediated currents (Bellingham et al., 1998; Taschenberger and von Gersdorff, 2000; Youssoufian et al., 2005; Steinert et al., 2010; Case et al., 2011; Ammer et al., 2012). Thus, our data are in line with other auditory nuclei. However, we showed electrophysiologically and pharmacologically that AMPARs in the mature MSO are mainly GluR2 free, contrasting other nuclei of the superior olivary complex (Joshi et al., 2004; Case et al., 2011; Felix and Magnusson, 2016; Lujan et al., 2019). The nearly complete absence of GluR2 subunits in mature MSO neurons may reflect a special need for EPSC speed or an activity-dependent source of calcium influx in adult MSO neurons. Indeed, here we demonstrate that synaptically evoked local calcium signals in adult MSO neurons are AMPAR- but not NMDAR-mediated, and that such signals occur under physiological conditions. Moreover, compared with the spatial restriction of calcium signals evoked by action potentials, synaptically evoked calcium transients persisted throughout the dendrite.

The developmental refinement in the excitatory synaptic physiology is paralleled by a structural rearrangement of glutamatergic inputs. From small synaptic dots scattered in the neuropil, glutamatergic inputs become larger and aligned with the dendrite and soma. The adult staining pattern of large en-passant terminals agrees with electron-microscopy evidence (Clark, 1969; Lindsey, 1975; Brunso-Bechtold et al., 1990).

\section{Relevance of the loss of global and the persistence of local calcium signals}

The developmental loss of dendritic calcium signals evoked by somatic action potentials and the persistence of local synaptic calcium influx throughout the dendrite indicate a switch from global and local calcium integration to a more localized form of 
calcium signaling. Furthermore, the main endogenous calcium buffer parvalbumin indicates that the local calcium influx through synaptic AMPARs remains spatially restricted. The rapid unbinding of calcium from parvalbumin will allow the extrusion mechanism to clear calcium locally from the influx site. The developmental switch from global to local calcium signaling could have general implications for plasticity. Since the integration of both types of calcium signals is known to trigger long-term or spike timing-dependent plasticity (Kampa et al., 2006; Zhao et al., 2006; Winters and Golding, 2018), the loss of global calcium signaling indicates the developmental time point at which such forms of synaptic plasticity are lost. Our data therefore highlight the potential time window for such forms of calcium- and activity-dependent plasticity after hearing onset. From this time window, it can be speculated that only a brief $2 \mathrm{~d}$ period exists after hearing onset during which acoustic activity can trigger calcium- and activity-dependent plasticity.

As shown here, calcium influx is very limited in adult MSO neurons. Other sources of calcium influx might be relevant for the full calcium homeostasis in MSO neurons, such as nonselective cation channels that were described in other auditory nuclei (Ene et al., 2003, 2007; González-Inchauspe et al., 2017). The only source of calcium influx, however, which can be correlated with activity, is via synaptic glutamate receptors. Thus, MSO neurons may require synaptic inputs to maintain a functional calcium homeostasis, for which the background activity arriving from the cochlear nuclei is essential.

\section{Mechanisms for developmental refinements in the MSO}

OWN rearing affects auditory processing in at least two ways. First, the activity levels of auditory neurons are expected to be raised due to the constant noise. Second, the directionality of spatial cues is compromised (Withington-Wray et al., 1990), and their instructing role in circuit formation is hampered (WithingtonWray et al., 1990; Kapfer et al., 2002; Magnusson et al., 2005; Seidl and Grothe, 2005). With this paradigm, we therefore alter both activity levels and the experience of localization, whereas genetically imprinted developmental programs should be unaffected. Thus, this method allows for at least a partial segregation of cues that drive developmental refinements.

Here, we describe an acceleration of calcium signaling and the excitatory synaptic refinement during late postnatal development upon OWN rearing. We favor the interpretation that raised activity levels induced by OWN accelerate the development of these cellular features. Since disrupting localization cues leads to a slowing or an arrest in the development of inhibitory inputs in this circuit (Kapfer et al., 2002; Magnusson et al., 2005; Seidl and Grothe, 2005), the observed acceleration might be based on increased overall activity levels. This interpretation is consistent with work showing that a loss of activity slows or arrests the development of excitatory inputs in the lateral superior olive (Kotak and Sanes, 1997). Thus, activity appears crucial to establish the framework of a neuronal coincidence detector circuit to promote its general output generation.

Together with previous studies, our findings give insight into which cues differentially drive developmental refinements in the MSO. The development of excitatory inputs, the source for output generation, is driven by activity levels. Inhibition, which is crucial for adjusting the temporal precision of this circuit to perform its task in ITD processing (Brand et al., 2002; Pecka et al., 2008; Goldwyn et al., 2017), requires the experience of specific spatial cues (Kapfer et al., 2002; Magnusson et al., 2005; Seidl and Grothe, 2005). Since biophysical properties, such as the mem- brane resistance, membrane time constant, action potential size, and after-hyperpolarization were unaffected by OWN exposure, we speculate that these are in large part regulated by genetic programs. Hence, each part of the MSO cell physiology might be instructed by a different cue during development.

How cellular signals triggered by these different regulatory cues interact remains unclear. Since the accelerated loss of calcium influx and the disruption of inhibitory signaling (Kapfer et al., 2002; Magnusson et al., 2005) were triggered by the same experimental paradigm, they are expected to lead to the same demonstrated breakdown of ITD tuning in the dorsal nucleus of the lateral lemniscus (Seidl and Grothe, 2005). Thus, as indicated before, the existence of an interaction between the refinement of inhibition and NMDARs (Winters and Golding, 2018) might be based on activity-evoked calcium influx. In this scenario, the accelerated loss of activity-driven calcium influx by action potentials and EPSCs may leave too little calcium signaling after hearing onset to guide the rapid, experience-dependent inhibitory refinement. Thus, it appears that cross-signaling between the two input types is crucial to establish the required synaptic balance for proper ITD function.

\section{References}

Ammer JJ, Grothe B, Felmy F (2012) Late postnatal development of intrinsic and synaptic properties promotes fast and precise signaling in the dorsal nucleus of the lateral lemniscus. J Neurophysiol 107:1172-1185.

Autret L, Mechaly I, Scamps F, Valmier J, Lory P, Desmadryl G (2005) The involvement of Cav3.2/alpha1H T-type calcium channels in excitability of mouse embryonic primary vestibular neurones. J Physiol 567: 67-78.

Bazwinsky-Wutschke I, Härtig W, Kretzschmar R, Rübsamen R (2016) Differential morphology of the superior olivary complex of Meriones unguiculatus and Monodelphis domestica revealed by calcium-binding proteins. Brain Struct Funct 221:4505-4523.

Bellingham MC, Lim R, Walmsley B (1998) Developmental changes in EPSC quantal size and quantal content at a central glutamatergic synapse in rat. J Physiol 511:861-869.

Berridge MJ (1998) Neuronal calcium signaling. Neuron 21:13-26.

Bowie D, Mayer ML (1995) Inward rectification of both AMPA and kainate subtype glutamate receptors generated by polyamine-mediated ionchannel block. Neuron 15:453-462.

Brand A, Behrend O, Marquardt T, McAlpine D, Grothe B (2002) Precise inhibition is essential for microsecond interaural time difference coding. Nature 417:543-547.

Brunso-Bechtold JK, Henkel CK, Linville C (1990) Synaptic organization in the adult ferret medial superior olive. J Comp Neurol 294:389-398.

Burnashev N, Monyer H, Seeburg PH, Sakmann B (1992) Divalent ion permeability of AMPA receptor channels is dominated by the edited form of a single subunit. Neuron 8:189-198.

Caicedo A, d'Aldin C, Puel JL, Eybalin M (1996) Distribution of calciumbinding protein immunoreactivities in the guinea pig auditory brainstem. Anat Embryol 194:465-487.

Case DT, Zhao X, Gillespie DC (2011) Functional refinement in the projection from ventral cochlear nucleus to lateral superior olive precedes hearing onset in rat. PLoS One 6:e20756.

Chambard JM, Chabbert C, Sans A, Desmadryl G (1999) Developmental changes in low and high voltage-activated calcium currents in acutely isolated mouse vestibular neurons. J Physiol 518:141-149.

Chirila FV, Rowland KC, Thompson JM, Spirou GA (2007) Development of gerbil medial superior olive: integration of temporally delayed excitation and inhibition at physiological temperature. J Physiol 584:167-190.

Clark GM (1969) Ultrastructure of nerve endings in medial superior olive of cat. Brain Res 14:293-305.

Couchman K, Grothe B, Felmy F (2010) Medial superior olivary neurons receive surprisingly few excitatory and inhibitory inputs with balanced strength and short-term dynamics. J Neurosci 30:17111-17121.

Cueni L, Canepari M, Adelman JP, Lüthi A (2009) $\mathrm{Ca}(2+)$ signaling by T-type $\mathrm{Ca}(2+)$ channels in neurons. Pflugers Arch 457:1161-1172. 
Doughty JM, Barnes-Davies M, Rusznák Z, Harasztosi C, Forsythe ID (1998) Contrasting $\mathrm{Ca}^{2+}$ channel subtypes at cell bodies and synaptic terminals of rat anterioventral cochlear bushy neurones. J Physiol 512:365-376.

Ene FA, Kullmann PH, Gillespie DC, Kandler K (2003) Glutamatergic calcium responses in the developing lateral superior olive: receptor types and their specific activation by synaptic activity patterns. J Neurophysiol 90:2581-2591.

Ene FA, Kalmbach A, Kandler K (2007) Metabotropic glutamate receptors in the lateral superior olive activate TRP-like channels: age- and experiencedependent regulation. J Neurophysiol 97:3365-3375.

Feldman DE (2012) The spike-timing dependence of plasticity. Neuron 75:556-571

Felix RA 2nd, Magnusson AK (2016) Development of excitatory synaptic transmission to the superior paraolivary and lateral superior olivary nuclei optimizes differential decoding strategies. Neuroscience 334:1-12.

Felmy F, Schneggenburger R (2004) Developmental expression of the $\mathrm{Ca}^{2+}$-binding proteins calretinin and parvalbumin at the calyx of held of rats and mice. Eur J Neurosci 20:1473-1482.

Franklin JL, Johnson EM Jr (1992) Suppression of programmed neuronal death by sustained elevation of cytoplasmic calcium. Trends Neurosci 15:501-508.

Futai K, Okada M, Matsuyama K, Takahashi T (2001) High-fidelity transmission acquired via a developmental decrease in NMDA receptor expression at an auditory synapse. J Neurosci 21:3342-3349.

Geiger JR, Melcher T, Koh DS, Sakmann B, Seeburg PH, Jonas P, Monyer H (1995) Relative abundance of subunit messenger-RNAs determines gating and $\mathrm{Ca}^{2+}$ permeability of AMPA receptors in principal neurons and interneurons in rat CNS. Neuron 15:193-204.

Goldwyn JH, McLaughlin M, Verschooten E, Joris PX, Rinzel J (2017) Signatures of somatic inhibition and dendritic excitation in auditory brainstem field potentials. J Neurosci 37:10451-10467.

González-Inchauspe C, Urbano FJ, Di Guilmi MN, Uchitel OD (2017) Acid-sensing ion channels activated by evoked released protons modulate synaptic transmission at the mouse calyx of held synapse. J Neurosci 37:2589-2599.

Greer PL, Greenberg ME (2008) From synapse to nucleus: calciumdependent gene transcription in the control of synapse development and function. Neuron 59:846-860.

Grothe B, Pecka M, McAlpine D (2010) Mechanisms of sound localization in mammals. Physiol Rev 90:983-1012.

Gu X, Spitzer NC (1993) Low-threshold $\mathrm{Ca}^{2+}$ current and its role in spontaneous elevations of intracellular $\mathrm{Ca}^{2+}$ in developing Xenopus neurons. J Neurosci 13:4936-4948.

Harasztosi C, Forsythe ID, Szucs G, Stanfield PR, Rusznák Z (1999) Possible modulatory role of voltage-activated $\mathrm{Ca}(2+)$ currents determining the membrane properties of isolated pyramidal neurones of the rat dorsal cochlear nucleus. Brain Res 839:109-119.

Hirtz JJ, Braun N, Griesemer D, Hannes C, Janz K, Löhrke S, Müller B, Friauf E (2012) Synaptic refinement of an inhibitory topographic map in the auditory brainstem requires functional Cav1.3 calcium channels. J Neurosci 32:14602-14616.

Hollmann M, Hartley M, Heinemann S (1991) $\mathrm{Ca}^{2+}$ permeability of KAAMPA-gated glutamate receptor channels depends on subunit composition. Science 252:851-853.

Iwasaki S, Takahashi T (2001) Developmental regulation of transmitter release at the calyx of held in rat auditory brainstem. J Physiol 534:861-871.

Joshi I, Wang LY (2002) Developmental profiles of glutamate receptors and synaptic transmission at a single synapse in the mouse auditory brainstem. J Physiol 540:861-873.

Joshi I, Shokralla S, Titis P, Wang LY (2004) The role of AMPA receptor gating in the development of high-fidelity neurotransmission at the calyx of held synapse. J Neurosci 24:183-196.

Kamboj SK, Swanson GT, Cull-Candy SG (1995) Intracellular spermine confers rectification on rat calcium-permeable AMPA and kainate receptors. J Physiol 486:297-303.

Kampa BM, Letzkus JJ, Stuart GJ (2006) Requirement of dendritic calcium spikes for induction of spike-timing-dependent synaptic plasticity. J Physiol 574:283-290.

Kapfer C, Seidl AH, Schweizer H, Grothe B (2002) Experience-dependent refinement of inhibitory inputs to auditory coincidence-detector neurons. Nat Neurosci 5:247-253.
Khurana S, Liu Z, Lewis AS, Rosa K, Chetkovich D, Golding NL (2012) An essential role for modulation of hyperpolarization-activated current in the development of binaural temporal precision. J Neurosci 32:28142823.

Koh DS, Burnashev N, Jonas P (1995) Block of native Ca(2+)-permeable AMPA receptors in rat brain by intracellular polyamines generates double rectification. J Physiol 486:305-312.

Kotak VC, Sanes DH (1997) Deafferentation weakens excitatory synapses in the developing central auditory system. Eur J Neurosci 9:2340-2347.

Kullmann PH, Kandler K (2008) Dendritic $\mathrm{Ca}^{2+}$ responses in neonatal lateral superior olive neurons elicited by glycinergic/GABAergic synapses and action potentials. Neuroscience 154:338-345.

Levic S, Nie L, Tuteja D, Harvey M, Sokolowski BH, Yamoah EN (2007) Development and regeneration of hair cells share common functional features. Proc Natl Acad Sci U S A 104:19108-19113.

Lindsey BG (1975) Fine-structure and distribution of axon terminals from cochlear nucleus on neurons in medial superior olivary nucleus of cat. J Comp Neurol 160:81-103.

Lohmann C, Friauf E (1996) Distribution of the calcium-binding proteins parvalbumin and calretinin in the auditory brainstem of adult and developing rats. J Comp Neurol 367:90-109.

Lohmann C, Ilic V, FriaufE (1998) Development of a topographically organized auditory network in slice culture is calcium dependent. J Neurobiol 34:97-112.

Lory P, Bidaud I, Chemin J (2006) T-type calcium channels in differentiation and proliferation. Cell Calcium 40:135-146.

Lujan B, Dagostin A, von Gersdorff H (2019) Presynaptic diversity revealed by $\mathrm{Ca}(2+)$-permeable AMPA receptors at the calyx of Held synapse. J Neurosci 39:2981-2994

Magee JC, Johnston D (1997) A synaptically controlled, associative signal for Hebbian plasticity in hippocampal neurons. Science 275:209-213.

Magnusson AK, Kapfer C, Grothe B, Koch U (2005) Maturation of glycinergic inhibition in the gerbil medial superior olive after hearing onset. J Physiol 568:497-512.

Markram H, Lübke J, Frotscher M, Sakmann B (1997) Regulation of synaptic efficacy by coincidence of postsynaptic APs and EPSPs. Science 275: 213-215.

Michaelsen K, Lohmann C (2010) Calcium dynamics at developing synapses: mechanisms and functions. Eur J Neurosci 32:218-223.

Myoga MH, Lehnert S, Leibold C, Felmy F, Grothe B (2014) Glycinergic inhibition tunes coincidence detection in the auditory brainstem. Nat Commun 5:3790.

Pecka M, Brand A, Behrend O, Grothe B (2008) Interaural time difference processing in the mammalian medial superior olive: the role of glycinergic inhibition. J Neurosci 28:6914-6925.

Pilati N, Linley DM, Selvaskandan H, Uchitel O, Hennig MH, KoppScheinpflug C, Forsythe ID (2016) Acoustic trauma slows AMPA receptor-mediated EPSCs in the auditory brainstem, reducing GluA4 subunit expression as a mechanism to rescue binaural function. J Physiol 594:3683-3703.

Rautenberg PL, Grothe B, Felmy F (2009) Quantification of the threedimensional morphology of coincidence detector neurons in the medial superior olive of gerbils during late postnatal development. J Comp Neurol 517:385-396.

Sanes DH (1993) The development of synaptic function and integration in the central auditory system. J Neurosci 13:2627-2637.

Sanes DH, Takács C (1993) Activity-dependent refinement of inhibitory connections. Eur J Neurosci 5:570-574.

Scheuss V, Bonhoeffer T (2014) Function of dendritic spines on hippocampal inhibitory neurons. Cereb Cortex 24:3142-3153.

Scott LL, Mathews PJ, Golding NL (2005) Posthearing developmental refinement of temporal processing in principal neurons of the medial superior olive. J Neurosci 25:7887-7895.

Seidl AH, Grothe B (2005) Development of sound localization mechanisms in the mongolian gerbil is shaped by early acoustic experience. J Neurophysiol 94:1028-1036.

Smith AJ, Owens S, Forsythe ID (2000) Characterisation of inhibitory and excitatory postsynaptic currents of the rat medial superior olive. J Physiol 529:681-698.

Spitzer NC, Lautermilch NJ, Smith RD, Gomez TM (2000) Coding of neuronal differentiation by calcium transients. BioEssays 22:811-817. 
Steinert JR, Postlethwaite M, Jordan MD, Chernova T, Robinson SW, Forsythe ID (2010) NMDAR-mediated EPSCs are maintained and accelerate in time course during maturation of mouse and rat auditory brainstem in vitro. J Physiol 588:447-463.

Taschenberger H, von Gersdorff H (2000) Fine-tuning an auditory synapse for speed and fidelity: developmental changes in presynaptic waveform, EPSC kinetics, and synaptic plasticity. J Neurosci 20: 9162-9173.

Werthat F, Alexandrova O, Grothe B, Koch U (2008) Experience-dependent refinement of the inhibitory axons projecting to the medial superior olive. Dev Neurobiol 68:1454-1462.

Winters BD, Golding NL (2018) Glycinergic inhibitory plasticity in binaural neurons is cumulative and gated by developmental changes in action potential backpropagation. Neuron 98:166-178.e2.

Withington-Wray DJ, Binns KE, Dhanjal SS, Brickley SG, Keating MJ (1990) The maturation of the superior collicular map of auditory space in the guinea pig is disrupted by developmental auditory deprivation. Eur J Neurosci 2:693-703.

Youssoufian M, Oleskevich S, Walmsley B (2005) Development of a robust central auditory synapse in congenital deafness. J Neurophysiol 94:3168-3180.

Zhao JP, Phillips MA, Constantine-Paton M (2006) Long-term potentiation in the juvenile superior colliculus requires simultaneous activation of NMDA receptors and L-type $\mathrm{Ca}^{2+}$ channels and reflects addition of newly functional synapses. J Neurosci 26:12647-12655. 